\title{
DIFFERENTIABLE FUNCTIONS WITH BOUNDED NONEMPTY SUPPORT ON BANACH SPACES
}

\author{
BY J. H. M. WHITFIELD ${ }^{1,2}$
}

Communicated by W. Rudin, September 14, 1965

1. Introduction. In [3, p. 26], S. Lang has raised the question whether or not a Banach space admits Fréchet differentiable partitions of unity. G. Restrepo [4] has shown that a Banach space with separable dual admits $C^{1}$ partitions of unity. In this note results are given which imply that, if the density character of a Banach space is strictly less than the density character of its dual, then the space does not admit Fréchet differentiable partitions of unity. This is an announcement of the results; detailed proofs will appear later.

2. Preliminaries. Let $X$ be a Banach space with norm $\rho$; let $S_{\rho}=\{x: x \in X \& \rho(x)=1\} . X$ will be said to admit a norm $\bar{\rho}$, if $\bar{\rho}$ is equivalent to $\rho$. The density character of $X$, denoted dens $X$, is the greatest lower bound of the cardinal numbers of dense subsets of $X$, or equivalently, the least upper bound of the cardinal numbers of discrete subsets of $X$. For $x, u \in X$, let

$$
\left(\rho^{\prime} x\right)(u)=\lim _{t \rightarrow 0^{+}} \frac{\rho(x+t u)-\rho(x)}{t}
$$

(this limit always exists). The support of a real or vector valued function $f$ on $X$ is the closure of the set $\{x: x \in X \& f(x) \neq 0\}$. Throughout this note differentiable will mean Fréchet differentiable.

3. Results. The main result is

THEOREM 1. If $X$ admits a norm $\bar{\rho}$ such that $\bar{\rho}^{\prime}$ is uniformly discontinuous (i.e., there exists $\epsilon>0$ such that for all $x \in X$ and $\delta>0$, there are $x_{1}, x_{2} \in X$ and $u \in S_{\bar{\rho}}$ such that $\bar{\rho}\left(x_{1}-x\right)<\delta, \bar{\rho}\left(x_{2}-x\right)<\delta$ and $\mid\left(\bar{\rho}^{\prime} x_{1}\right)(u)$ $\left.-\left(\bar{\rho}^{\prime} x_{2}\right)(u) \mid>\epsilon\right)$, then there exists no differentiable real valued function on $X$ with bounded nonempty support.

The proof of Theorem 1 uses methods similar to those of J. Kurzweil [2], who essentially proved the above result for the spaces $l_{1}$ and $C[0,1]$ and continuously differentiable functions. R. Bonic and

1 This research was supported in part by a grant from the Case Fund.

2 The results announced here are contained in the author's doctoral thesis. The author wishes to express his thanks to Professor E. B. Leach for his guidance throughout this research. 
J. Frampton [1] removed the continuity condition on the differential from Kurzweil's hypothesis.

Theorem 2. If dens $X<$ dens $X^{*}$, then $X$ admits a norm $\bar{\rho}$ such that $\bar{\rho}^{\prime}$ is uniformly discontinuous.

4. Remarks. It should also be noted that dens $X<$ dens $X^{*}$ implies that there are no differentiable functions with bounded nonempty support from $X$ into any Banach space $Y$. For, if $f: X \rightarrow Y$ is differentiable and has bounded nonempty support, then there exists $g \in Y^{*}$ such that $g \circ f$ has bounded nonempty support (which is certainly contained in the support of $f$ ). Further, if any subspace of $X$ satisfies the hypothesis of Theorem 1, then the result is still true. Moreover, if $X$ is the cartesian product of $Y$ and another space, and if $Y$ admits a norm $\bar{\rho}$ such that $\bar{\rho}^{\prime}$ is uniformly discontinuous, then $X$ has the same property. Finally, it may be observed that $X$ can be chosen to be $Y \times Z$ where dens $Y<$ dens $Y^{*}=\operatorname{dens} Z=\operatorname{dens} Z^{*}$, so that dens $X$ $=$ dens $X^{*}$. This shows that dens $X=\operatorname{dens} X^{*}$ does not imply the existence of a real valued differentiable function on $X$ with bounded nonempty support.

\section{BIBLIOGRAPHY}

1. R. Bonic and J. Frampton, Differentiable functions on certain Banach spaces, Bull. Amer. Math. Soc. 71 (1965), 393-395.

2. J. Kurzweil, On approximation in real Banach spaces, Studia Math. 14 (1954), 213-231.

3. S. Lang, Introduction to differentiable manifolds, Interscience, New York, 1962.

4. G. Restrepo, Differentiable norms in Banach spaces, Bull. Amer. Math. Soc. 70 (1964), 413-414.

Case Institute of Technology 